\title{
Manual Respiratory Therapy Device in Subacute COVID-19: A Case Report
}

\section{Duygu Zorlu*}

*Correspondence: Duygu Zorlu

Address: Department of Respiratory Diseases, Faculty of Medicine, Ahi Evran University, Kirsehir, Turkey e-mail $\bowtie:$ trbzorlu@yahoo.com

Received: 28 July 2021; Accepted: 05 August 2021

Copyright: (C) 2021 Zorlu D. This is an open-access article distributed under the terms of the Creative Commons Attribution License, which permits unrestricted use, distribution, and reproduction in any medium, provided that the original work is properly cited.

\section{ABSTRACT}

\begin{abstract}
There are no recommendations for medical therapy or auxiliary breathing device at an evidence level to prevent symptoms at subacute phase of novel coronavirus-2019 (COVID-19). As patients are usually symptomatic, they have difficulty in performing their daily activities.

A 38-year-old male patient working as a medical secretary was admitted with a complaint of tickle in throat. The reverse transcriptase-polymerase chain reaction test was positive. Under treatment, the patient got worsed. He was hospitalized and respiratory failure was seen. He transferred to the intensive care unit (ICU). In the follow-ups, the patient who was taken to the service from the ICU and then discharged was followed up routinely. Than during the follow-up in the outpatient setting, the patient started to use auxiliary breathing device. At one month, he had no complaint other than dyspnea on exertion, and pulmonary function parameters completely improved.

Therefore, this case highlights the importance of using auxiliary breathing device which can yield benefits during the subacute phase of COVID-19.
\end{abstract}

Keywords: COVID-19, Auxiliary Breathing Device, Postural Drainage, Airways

\section{Introduction}

The first novel coronavirus-2019 (COVID-19) case caused by severe acute respiratory syndromecoronavirus 2 (SARS-CoV-2) was identified in the Wuhan, Hubei province of China in December 2019 and rapidly spread to the whole world, and the World Health Organization (WHO) declared COVID-19 pandemic on March 11th, 2020 (Zhu et al., 2020) [1]. The first COVID-19 case in Turkey was diagnosed on March 11th, 2020 and the first virus-related death was reported on March 15th, 2020 (Zhu et al., 2020) [1]. On April 1st, 2020, the Republic of Turkey, Ministry of Health announced that COVID-19 spread across the country [2].

From the beginning of the pandemic, global research efforts have been focused on this deadly virus with high pathogenicity. During this period, although our knowledge and experiences on the virus have been increasing day by day, there is still no therapeutic option at an evidence level, yet. (We can add the sentence that the reviewer suggested to add about antıbodies.) 
In about the 10th month of the pandemic, clinicians have gained many experiences about patients and disease course. The most common symptoms of COVID-19 include dry cough and/or sputum production, shortness of breath, and malaise-fatigue complaints at any time after the disease. In addition, symptoms such as loss of taste and smell, widespread muscle and joint pain, and diarrhea are also observed (Guan et al., 2020; Huang et al., 2020). These patients are usually followed under inhaled therapy, anticoagulants, and symptomatic treatment (Huang et al., 2020).

Based on our increasing experience, auxiliary breathing device may be beneficial in these patients. In this article, we present a case of COVID-19 who received auxiliary breathing device and discuss symptom prevention and pulmonary improvement without sequelae in the light of literature data.

\section{Case Report}

A 38-year-old male patient working as a medical secretary was admitted with a complaint of tickle in throat. The reverse transcriptase-polymerase chain reaction (RT-PCR) test was immediately performed, as the patient was in the high-risk occupational group. The test result was positive and the patient was referred to our clinic. On physical examination, he had no additional symptoms with normal vital signs, routine biochemistry values, and thoracic computed tomography (CT) findings. The treatment recommended in the Republic of Turkey, Ministry of Health algorithm (hydroxychloroquine $200 \mathrm{mg} /$ day, b.i.d. and favipiravir 1,600 mg/day loading dose, b.i.d., followed by $600 \mathrm{mg} /$ day maintenance dose, b.i.d.) and low-molecular-weight heparin (LMWH) was initiated, and the patient went into home quarantine. He was isolated at home with regular treatment. Daily phone calls were made, symptoms were questioned, and he was questioned to evaluate patient compliance to treatment. On day 3 , he suffered from cough and oropharyngeal mouthwash and short-acting beta-2 agonist were initiated. On day 5 of his treatment, cough became more pronounced and the patient was scheduled for evaluation in the outpatient setting. His vital signs were as follows: oxygen saturation (SpO2) 92\% and fever 37.5oC. Since his symptoms worsened during treatment, the patient was hospitalized. Routine laboratory test results were as follows: lymphocyte count $0.7610^{\wedge} 3 / \mathrm{uL}(19.4 \%)$, monocyte count $0.2710^{\wedge} 3 / \mathrm{uL}$, white blood cell (WBC) count $3.410^{\wedge} 3 / \mathrm{uL}$, procalcitonin indicating a low risk for sepsis, CRP $1.4 \mathrm{mg} / \mathrm{dL}$, platelet $14710^{\wedge} 3 / \mathrm{UL}$, and magnesium $1.6 \mathrm{mg} / \mathrm{dL}$. Ferritin, cardiac markers, D-dimer, vitamin B12, and folate levels were within the normal range. As expectedly, thoracic CT scan showed mild-to-moderate COVID-19 pneumonia. It was decided to extend the current treatment to 10 days. In addition to the treatment, steroid $60 \mathrm{mg}$ and nebulizer treatment were initiated [3]. As his fever persisted after 72 hours of treatment, intravenous moxifloxacin was added to the treatment.

Despite medical treatment, the patient developed shortness of breath within two days and his 
cough worsened. Even, he had difficulty in talking. During follow-up, Sp02\% value decreased to 72\% with the increasing severity of dyspnea. In the laboratory tests, lymphocyte count was $0.6710^{\wedge} 3 / \mathrm{UL}$, monocyte count was $0.2710^{\wedge} 3 / \mathrm{UL}$, platelet count was $13110^{\wedge} 3 / \mathrm{UL}$, and CRP was $1.4 \mathrm{mg} / \mathrm{dL}$. Repeated thoracic CT revealed mild-to-moderate COVID-19 pneumonia (Fig. 1a, b, c, d).

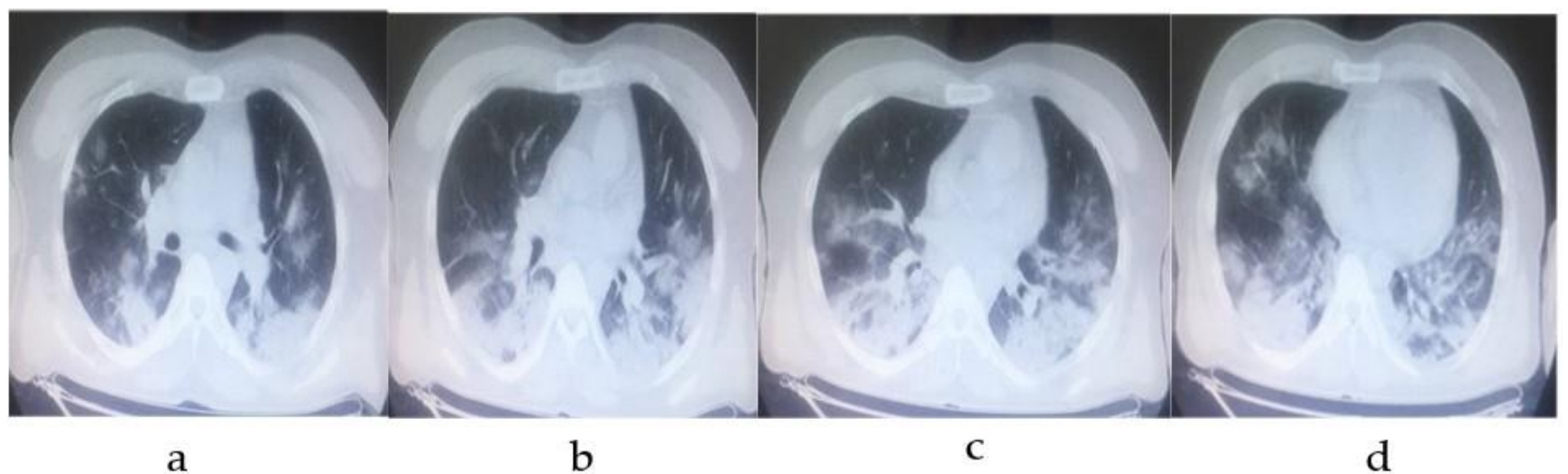

Figure 1 (a, b, c, d): Thoracic computed tomography images at the time of intensive care unit stay showing.

The patient was referred to the intensive care unit (ICU) and intermittent continuous positive airway pressure (CPAP) support was immediately initiated. The patient was placed in the prone position at regular intervals. He was given steroid treatment from the first day of hospitalization, and tocilizumab $400 \mathrm{mg} /$ day b.i.d. added [3]. During the next two days, the patient was dyspneic and, despite using CPAP and $10 \mathrm{~L} / \mathrm{min}$ oxygen support, the Sp02\% was about 93\%. In routine laboratory tests, the lymphocyte count was $0.6610^{\wedge} 3 / \mathrm{UL}$, monocyte count was $0.0610^{\wedge} 3 / \mathrm{UL}$, platelet count was $29410^{\wedge} 3 / \mathrm{UL}$, and CRP was $0.2 \mathrm{mg} / \mathrm{dL}$. On day 3 of ICU hospitalization, the SpO2\% value started to slowly increase with the current treatment. The patient started to breathe relatively easier only with oxygen support. Intermittent CPAP use was continued. During the next days, dyspnea and cough symptoms started to substantially regress. Under the prespecified treatment, the patient was followed in the ICU for seven days in intermittent prone position and using CPAP, steroid, and inhaled therapy. At the end of day 7, the SpO2\% value increased to $96 \%$ using only $2 \mathrm{~L} / \mathrm{min}$ nasal oxygen support. In routine labor atory tests, lymphocyte count was $1.0010^{\wedge} 3 / \mathrm{UL}$, monocyte count was $0.2910^{\wedge} 3$ /UL, platelet count was $23610^{\wedge} 3 / \mathrm{UL}$, and CRP was $0.1 \mathrm{mg} / \mathrm{dL}$. The patient was transferred to the inpatient ward. Control thoracic CT showed severe viral pneumonia (Fig. 2a, b, c, d). During the inpatient ward stay, current medical treatment protocol was continued. Without the need for CPAP support, the patient was followed using oxygen (2 to $3 \mathrm{~L} / \mathrm{min})$ at intermittent prone position. He became better with each passing day. His dyspnea reduced and he had no cough. In routine laboratory tests, the lymphocyte count was $1.6810^{\wedge} 3 / \mathrm{UL}$, monocyte count was 0.63 $10^{\wedge} 3$ /UL, platelet count was $26010^{\wedge} 3 / \mathrm{UL}$, CRP was $0 \mathrm{mg} / \mathrm{dL}$, and WBC was $8.7810^{\wedge} 3 / \mathrm{uL}$. Repeated thoracic CT revealed generalized ground-glass consolidation areas and patchy subsegmental atelectasis, 
compatible with severe viral pneumonia (Fig. 3a, b, c, d). The patient no longer needed at-home oxygen support, and Sp02\% value increased to 96\% without oxygen support. The patient was followed in the inpatient ward for 10 more days and, then, discharged with recommendations for staying in the prone position at regular intervals and using inhaled therapy, low-dose steroid, and low-molecular-weight heparin (LMWH). His prescription was arranged, and the patient was scheduled for follow-up in the outpatient setting after 10 days.

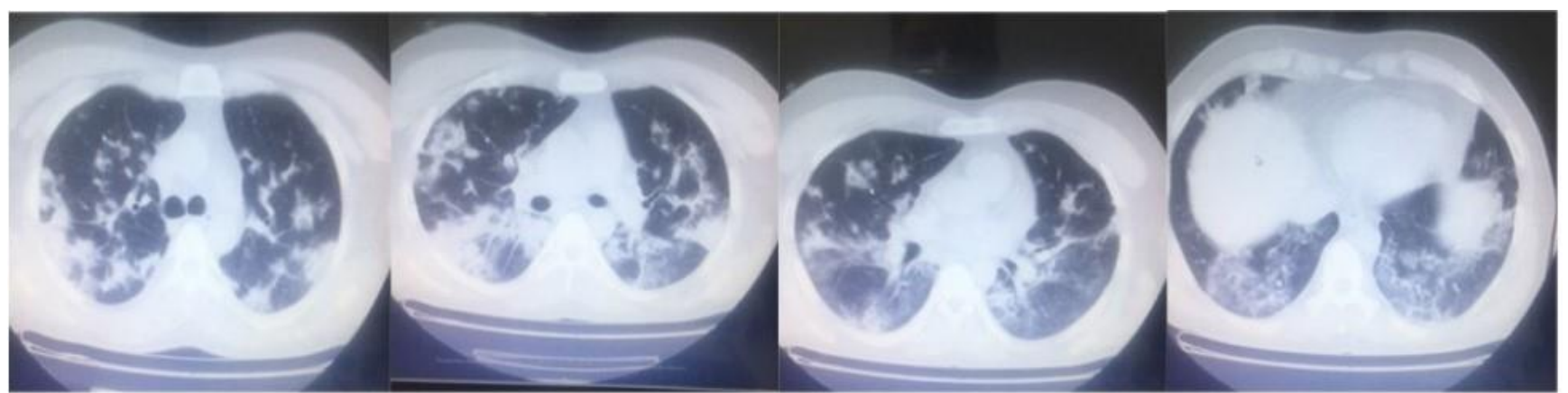

a

b

C

d

Figure 2 (a, b, c, d): Thoracic computed tomography images at the time of inpatient ward stay showing.

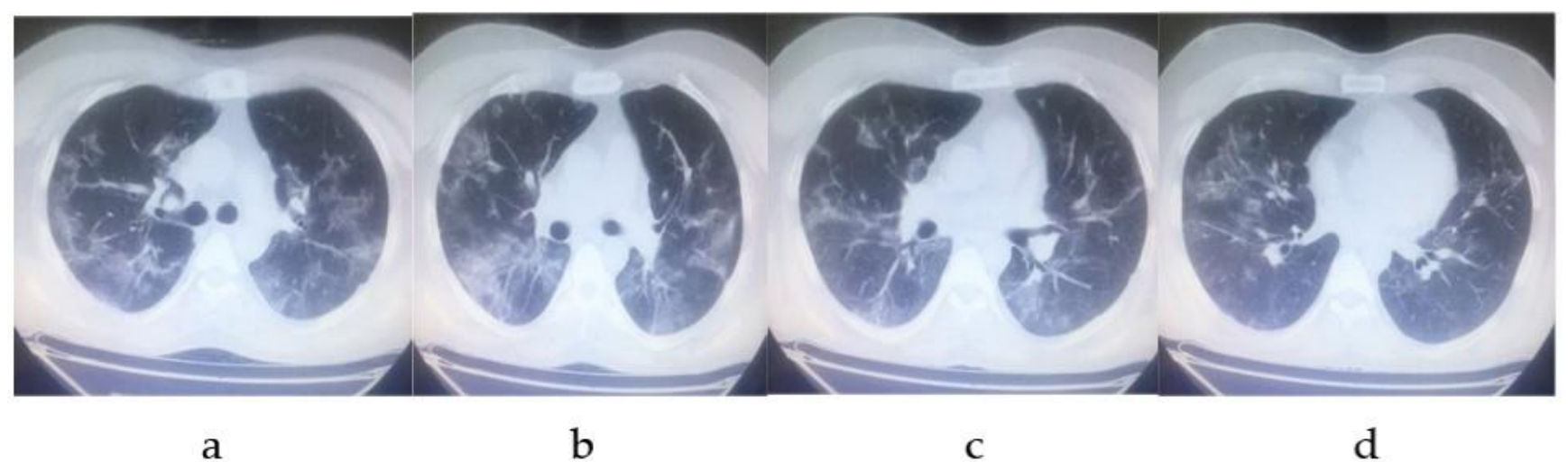

Figure $3(\mathbf{a}, \mathbf{b}, \mathbf{c}, \mathbf{d})$ : Thoracic computed tomography images at the time of inpatient ward stay immediately before discharge showing.

At the time of follow-up, he only had dyspnea with exertion. His blood parameters were within the normal range. The next follow-up was performed 15 days later and the patient's symptoms significantly improved and his routine blood parameters were within the normal range. Repeated thoracic CT, there was no ground-glass consolidation areas and patchy subsegmental atelectasis, it has neary normal radoologic findings (Fig. 4a, b, c, d). He was advised to continue his current treatment, and an auxiliary breathing device was prescribed (Fig. 5a, b). The device was initiated during the outpatient clinic followup, since the patient had dyspnea. As the patient was supported with medical therapy and his dyspnea was improved, he was able to make the necessary effort for device use. The patient underwent pulmonary function test (PFT) before device use (Fig. 6a). The patient was informed about the device use verbally 
and in written. Within the patient's knowledge, the cost of the device was covered by the investigators. The device use was explained to the patient with a detailed practice. Daily use schedule was given which was reminded by calling by phone. At one month of follow-up, his dyspnea with exertion was improved. The patient could expectorate easier and his shortness of breath apparently resolved. Routine blood parameters were normal, and pulmonary involvement regressed completely. Control thoracic CT showed usual radiological findings. At the end of one month of device use, control PFT was performed (Fig. 6b).

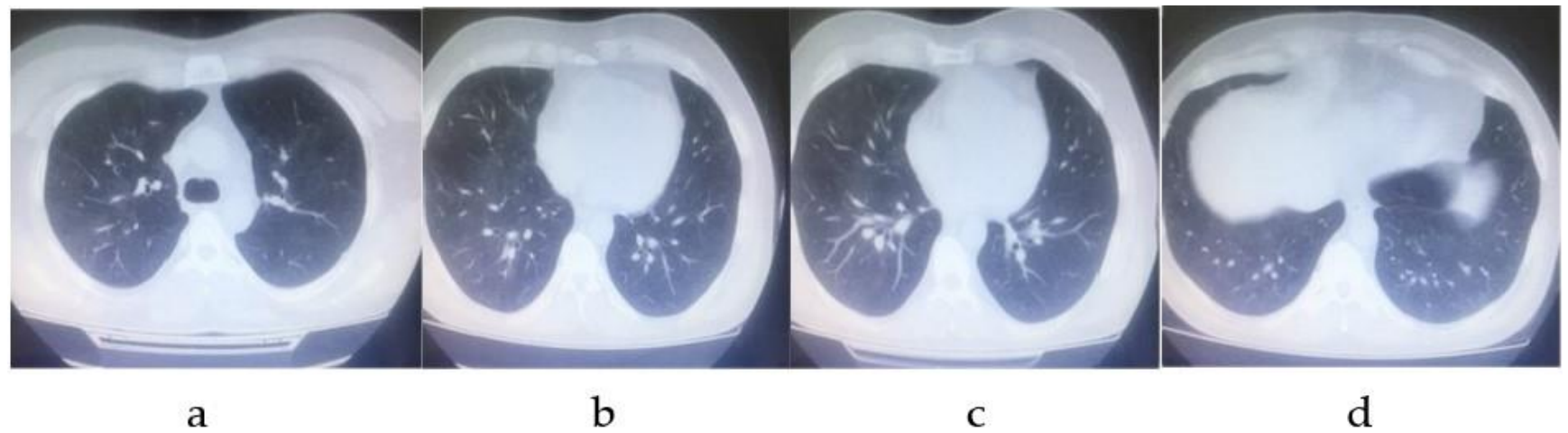

Figure 4 (a, b, c, d): Thoracic computed tomography images at one month after discharge showing.

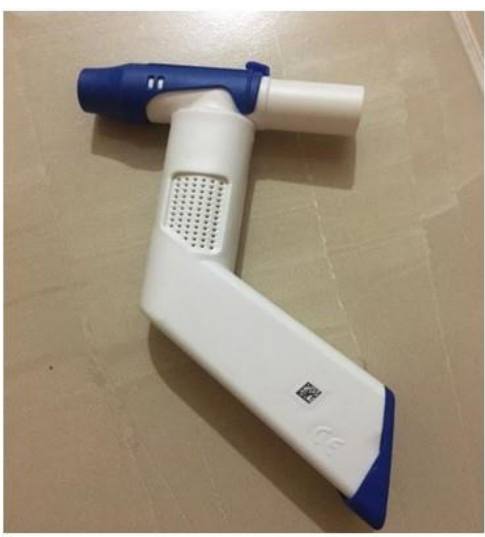

a

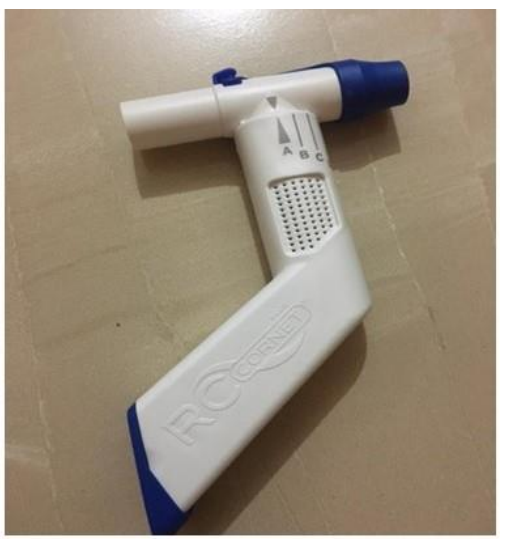

b

Figure 5: (a) Manual respiratory therapy device (b) Manual respiratory therapy device

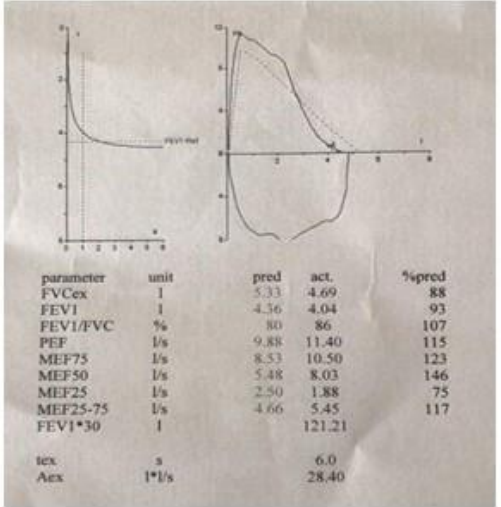

a

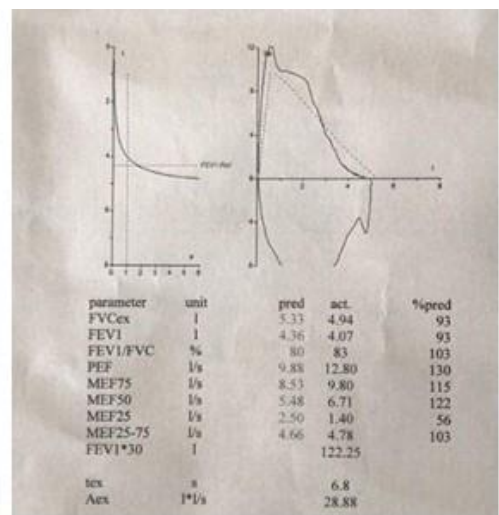

b 
Figure 6: (a) Pre-device pulmonary function test results (b) Post-device pulmonary function test results.

The patient is still under follow-up on a monthly basis in the outpatient setting and using longacting beta- 2 agonist and inhaled corticosteroid, anticoagulant, and auxiliary breathing device [3].

A written informed consent was obtained from the patient for all diagnostic and therapeutic procedures.

\section{Discussion}

The COVID-19 outbreak has rapidly affected all aspects of daily life throughout the world with varying degrees, leading to an unprecedented crisis. Although COVID-19 involves many tissues in the human body, the lungs are the main organs affected by the virus with persistent lung impairment in many patients even after months (Hui et al., 2005; Ngai et al., 2010; Park et al., 2018; Fleet et al., 2017).

The present case developed severe respiratory failure due to COVID-19, and all recommended treatment protocols were administered. The patient was discharged; however, his symptoms persisted during the subacute phase of COVID-19, despite somewhat improvement. Therefore, the patient was prescribed an auxiliary breathing device in addition to medical support. The main goals of using this device are to strengthen the peribronchial muscle tissue, reduce bronchial hyperreactivity, and facilitate expectoration, thereby, preventing the risk of secondary bacterial infections (Haidl et al., 2002). Although the long-term effects of this disease still remain to be elucidated, we believe that this device can reduce sequelae (i.e., respiratory tract pathologies) during the subacute phase of COVID-19.

According to the pre- and post-device PFT results, the increase in forced vital capacity (FVC), (FEV1), expiratory flow (PEF) in one second in forced expiratory volume indicates that the patient's perceived symptoms improve and they benefit from the device.

Apparently, this device was not available in studies on COVID-19 disease. However, there are one study on cystic fibrosis. Oscillatory positive expiratory pressure (OPEP) is an airway clearance therapy that delivers positive pressure and air-flow oscillations during exhalation. This study described functional characteristic differences of 4 OPEP devices during an active exhalation in a simulated model (Haidl et al., 2002; Van Fleet et al., 2017; Cegla et al., 2002). Resistance settings for the Acapella, RC-Cornet, Flutter, and Aerobika were adjusted to low, medium, and high. Values for f, Ppeak, PEP, and pressure amplitude were recorded for $1 \mathrm{~min}$ and graphically displayed. Statistically significant and clinically relevant variations in Ppeak, PEP, and pressure amplitude occurred between devices and within a device, as the resistance setting changed ( $\mathrm{Ni} \mathrm{Y}$ et al., 2018). The combination of device, time, and resistance settings 
affects OPEP device output for pressure, amplitude, and oscillatory frequency (Ni Y et al., 2018).

In a study with patients in the intensive care unit, OPEP exhibited a greater effectiveness in draining sputum, improving oxygenation and reducing inflammatory status in patients with lower respiratory tract infections compared with MP; however, it did not promote the elimination of microbes (Ni Y et al., 2018).

\section{Conclusion}

In conclusion, thanks to its ease of use, accessibility, safety, beneficial effects, and low cost, the use of an auxiliary breathing device seems to be reasonable and feasible in COVID-19 patients, both during the subacute and symptomatic phases. In cases where pulmonary embolism, thoracic trauma, or pneumothorax cannot be ruled out, there is no adverse effect of device use. Nonetheless, further multicenter, large-scale, long-term, randomized-controlled trials are needed to gain a better understanding of the use of these devices during the treatment and post-COVID period and even of their ability to prevent complications. With these promising results obtained from this case, we plan ongoing studies to gain more insight into this topic.

\section{References}

Cegla UH, Jost HJ, Harten A, Weber T, Wissmann S. Course of Severe COPD with and without Physiotherapy with the RCCornet(R). Pneumologie 200; 56: 418-24.

Fleet VH, Dunn DK, McNinch NL, Volsko TA. Evaluation of Functional Characteristics of 4 Oscillatory Positive Pressure Devices in a Simulated Cystic Fibrosis Model. Respir Care 2017; 62: 451-458.

Guan WJ, Ni ZY, Hu Y, Liang WH, Ou CQ, He JX, Liu L, Shan H, Lei CL, Hui DS, Du B. Clinical Characteristics of Coronavirus Disease 2019 in China. N Engl J Med 2020; 382: 1708-1720.

Haidl P, Rickert G, Cegla UH, Köhler D. Influence of an oscillating physiotherapy device (RC-Cornet trade mark) on the regional distribution of the pulmonary aerosol deposition in patients with COPD. Pneumologie 2002; 56: 498-502.

Huang C, Wang Y, Li X, Ren L, Zhao J, Hu Y, Zhang L, Fan G, Xu J, Gu X, Cheng Z. Clinical features of patients infected with 2019 coronavirus in Wuhan, China. Lancet 2020; 395: 497-506.

Hui DS, Joynt GM, Wong KT, Gomersall CD, Li TS, Antonio G, Ko FW, Chan MC, Chan DP, Tong MW, Rainer TH. Impact of severe acute respiratory syndrome (SARS) on pulmonary function, functional capacity and quality of life in a cohort of surviv ors. Thorax 2005; 60: 401-409.

Ngai JC, Ko FW, Ng SS, To KW, Tong M, Hui DS. The long-term impact of severe acute respiratory syndrome on pulmonary function, exercise capacity and health status. Respirology (Carlton, Vic) 2010; 15: 543-550.

Ni Y, Ding L, Yu Y, Dai R, Chen H, Shi G. Oscillatory positive expiratory pressure treatment in lower respiratory tract infection. Exp Ther Med 2018; 16: 3241-3248.

Park WB, Jun KI, Kim G, Choi JP, Rhee JY, Cheon S, Lee CH, Park JS, Kim Y, Joh JS, Chin BS. Correlation between Pneumonia 
Severity and Pulmonary Complications in Middle East Respiratory Syndrome. J Korean Med Sci 2018; 33 : e169.

Van Fleet H, Dunn DK, McNinch NK, Volsko TA. Evaluation of Functional Characteristics of 4 Oscillatory Positive Pressure Devices in a Simulated Cystic Fibrosis Model. Respir Care 2017; 62: 451-458.

Zhu N, Zhang D, Wang W, Li X, Yang B, Song J, Zhao X, Huang B, Shi W, Lu R, Niu P. A Novel Coronavirus from Patients with Pneumonia in China, 2019. N Engl J Med 2020; 382: 727-733.

1. https://www.worldometers.info/coronavirus/

2. https://www.who.int/emergencies/diseases/novel-coronavirus-2019/events-as-they-happen WHO 2020.

3. Rebuplic of Turkey Minıstry of Health COVID-19 Information page. COVID-19 Treatment. https://covid19.saglik.gov.tr/TR-66299/covid-19-tedavi.html. 